\section{Respiratory rates and freezing tolerance of embryos of Inga vera Willd. subsp. affinis (DC.) T.D. Penn. from different regions}

\author{
Edmir Vicente Lamarca ${ }^{1}$ (D), Claudio José Barbedo²*
}

ABSTRACT: Recalcitrant seeds are sensitive to desiccation and low storage temperatures, but there is a gradient of recalcitrance between different species and between populations of the same species. Therefore, tolerance to drying and low temperatures, as well as the respiratory rates of seeds, can be conditioned by the source of the material. The present study aimed to evaluate the relationship among desiccation and low temperature tolerance, and respiratory rates of Inga vera embryos from different regions. The embryos were submitted to three drying levels and incubated for up to fifteen days at $-4,-2,2$ and $5{ }^{\circ} \mathrm{C}$. Then, they were evaluated for respiration, germination and electrical conductivity. The embryos tolerated freezing at $-4{ }^{\circ} \mathrm{C}$ since they were associated with certain levels of drying and a consequent change in the energy status of water. In conclusion, the conditions in which the embryos are formed define their degree of maturation at dispersal, hence their tolerance to desiccation and freezing.

Index terms: Leguminosae, recalcitrant seeds, storage.

RESUMO: Sementes recalcitrantes são sensíveis à dessecação e às baixas temperaturas de armazenamento, mas há um gradiente de recalcitrância entre espécies diferentes e entre populações da mesma espécie. Portanto, a tolerância à secagem e às baixas temperaturas, bem como as taxas respiratórias de sementes podem ser condicionadas pela procedência do material. No presente trabalho objetivou-se avaliar a relação entre os níveis de secagem, a tolerância a baixas temperaturas e as taxas respiratórias de embriões de Inga vera procedentes de diferentes regiões de coleta. Os embriões foram submetidos a três níveis de secagem e incubados por até quinze dias a -4, -2, 2 e $5^{\circ} \mathrm{C}$. Em seguida, foram avaliados quanto à respiração, germinação e condutividade elétrica. Verificou-se a possibilidade de congelamento a $-4{ }^{\circ} \mathrm{C}$ desses embriões desde que associado a determinados níveis de secagem e consequente mudança no estado energético da água. Concluiu-se que as condições nas quais os embriões são formados condicionam seu grau de maturação na dispersão e sua tolerância à dessecação e ao congelamento.

Termos para indexação: Leguminosae, sementes recalcitrantes, armazenamento.
Journal of Seed Science, v.43, e202143017, 2021

http://dx.doi.org/10.1590/ 2317-1545v43247247
*Corresponding author E-mail: cjbarbedo@yahoo.com.br

Received: $1 / 5 / 2021$. Accepted: 5/27/2021.

\footnotetext{
${ }^{1}$ Universidade Ibirapuera, Avenida Interlagos, 1329, 04661-100 - São Paulo, SP, Brasil.

${ }^{2}$ Instituto de Botânica, Núcleo de Pesquisa em Sementes, Av. Miguel Stéfano, 3687, 04301-902 - São

Paulo, SP, Brasil.
} 


\section{INTRODUCTION}

The biggest current challenge faced by seed storage is to maintain the viability of seeds sensitive to desiccation for prolonged periods. As their water content cannot be reduced to values that substantially reduce their metabolism and that of associated microorganisms, the two major storage tools, namely drying and freezing, cannot be used for these seeds (FAO, 2014; Barbedo, 2018; Silva et al., 2018; Mayrink et al., 2019).

Sensitivity to seed desiccation is not a situation of absolute extremes, but a gradient that ranges from the most sensitive (or most recalcitrant) to the most tolerant (or most orthodox). It is also influenced by factors such as genetics, seed formation conditions and degree of maturation during seed dispersal (Daws et al., 2006; Walters et al., 2013; Walters, 2015; Barbedo, 2018).

Previous studies on the deterioration processes of these seeds, therefore, must take these factors into account, including materials from different origins, crops and degrees of maturity. Different responses with recalcitrant seeds of the same species have often been reported in the literature, e.g., those described for Inga vera Willd. subsp. affinis (A.D.) T.D. Pennington, whose seeds are among the most sensitive to desiccation and with the shortest longevity known to date (Bonjovani and Barbedo, 2008; Lamarca et al., 2013; Pereira et al., 2020).

Seed deterioration is inevitable, but its effects can be reduced by decreasing water content and temperature to reduce metabolic activity, with effects on the respiration rates of embryos (Marcos-Filho, 2015). Furthermore, seeds stored with high water contents are susceptible to damage caused by freezing temperatures, which can result in loss of viability (Bonjovani and Barbedo, 2008).

Insufficient knowledge about the factors involved in behavioral differences has been a major obstacle to storage and conservation of species with desiccation-sensitive seeds, especially in restoration programs and in germplasm banks (Pereira et al., 2020). Seeds of Inga vera subsp. affinis have proved to be an excellent tool for this type of research (Bilia et al., 2003), especially in studies on intolerance to desiccation and low temperatures, respiratory rates under different conditions, responses to the application of metabolism inhibitors, action of associated microorganisms, among other aspects. This is because they are extremely sensitive to desiccation and have interesting biochemical and physiological responses, such as variations in respiratory rates under different conditions (Andréo et al., 2006; Bonjovani and Barbedo, 2008; Caccere et al., 2013; Lamarca et al., 2013; Bonjovani and Barbedo, 2014; Lamarca and Barbedo, 2015; Parisi et al., 2016, 2019; Bonjovani and Barbedo, 2020; Pereira et al., 2020).

In the present study, assuming that environmental conditions are decisive for the maturation stage in which the seeds are dispersed and, consequently, for the characteristics of the embryos, the objective was to evaluate the relationship among drying levels, tolerance to low temperatures and the respiratory rates Inga vera embryos, by assessing embryos from different collection regions.

\section{MATERIAL AND METHODS}

Embryos of Inga vera subsp. affinis were collected from mature fruits (Andréo et al., 2006; Bonjovani and Barbedo, 2008) in January and February from matrices located in Ribeirão Vermelho (RIB), MG $\left(21^{\circ} 11^{\prime} \mathrm{S}, 45^{\circ} 03^{\prime} \mathrm{W}\right.$

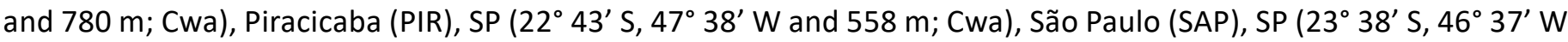
and $785 \mathrm{~m} ; \mathrm{Cwb}$ ) and Pariquera-Açu (PAR), SP (24. $37^{\prime} \mathrm{S}, 47^{\circ} 53^{\prime} \mathrm{W}$ and $28 \mathrm{~m}$; Af). Meteorological data for the period between maximum flowering and collection of ripe fruits were gathered at meteorological stations located close to the collection areas for calculation of cumulative rainfall $(\mathrm{mm})$ and degrees-day $\left({ }^{\circ} \mathrm{C} \mathrm{d}\right)$, the latter following equations proposed by Villa Nova et al. (1972), considering the base temperature of $10^{\circ} \mathrm{C}$. In the laboratory, the fruits were opened manually to remove the seeds, whose integument (sarcotesta, typical of seeds of species of the genus Inga - Braz et al., 2012) were removed to obtain the embryos. These were characterized in terms of water content, water potential, dry weight content, germination, electrical conductivity and respiration, as described below. 
Water content and dry weight content were determined gravimetrically by the oven method at $103{ }^{\circ} \mathrm{C}$ for seventeen hours, with results expressed on a wet basis (Brasil, 2009). Water potential (MPa) was measured in a WP4 potentiometer (Decagon Devices, Pullman), based on the dew point temperature of the air in equilibrium with the tested sample (Bonjovani and Barbedo, 2014). The germination test was carried out on a paper roll, in BOD chambers, model $347 \mathrm{CD}$ (Fanem, São Paulo), at $25{ }^{\circ} \mathrm{C}$ and continuous light. The evaluations were performed every two days for 36 days; data was recorded on the number of embryos that emitted primary roots (to calculate germination) and those that produced normal seedlings, and average germination time was calculated (Santana and Ranal, 2004; Guardia et al, 2020). The first primary root emitted was considered as germination; only one primary root unit was counted, since there was no occurrence of polyembryony.

Electrical conductivity (EC) was determined using a benchtop digital conductivity meter MA150 (Marconi, Piracicaba), calibrated to $146.7 \mu \mathrm{S} . \mathrm{cm}^{-1}$. For this purpose, ten embryos were placed in plastic cups $300 \mathrm{~mL}$, containing $75 \mathrm{~mL}$ of deionized water, and then incubated in BOD-type chambers at $20^{\circ} \mathrm{C}$, in the absence of light. The evaluations were made after 24 hours of incubation, and the results were calculated based on the dry weight of the sample, providing the conductivity per unit of dry weight ( $\mu \mathrm{S} . \mathrm{cm}^{-1} \cdot \mathrm{gDW}^{-1}$, according to Barbedo and Cicero, 1998).

The respiratory rates of embryos from Inga vera were evaluated using an Illinois 6600 gas analyzer (Illinois Instruments, Inc., Johnsburg), with embryos incubated in respirometers ( 0.6 L glass vials, hermetically sealed), in the absence of light, for five days, to determine the percentage by volume of $\mathrm{O}_{2}$ and $\mathrm{CO}_{2}$. This data was used to determine the rates of $\mathrm{O}_{2}$ consumption and $\mathrm{CO}_{2}$ release expressed in $\mu \mathrm{mol}$.gDW ${ }^{-1} \cdot \mathrm{d}^{-1}$ (Lamarca and Barbedo, 2012). The respiratory quotient ( $\mathrm{RQ}=\mathrm{CO}_{2}$ released $/ \mathrm{O}_{2}$ consumed) was calculated according to the equation described by Kader and Saltveit (2002). Supplementary evaluations were performed every five days, in a total of ten and fifteen days of incubation. Such evaluations were carried out on the same embryos, that is, an evaluation with five days of incubation, another with ten days and another with fifteen days of incubation; the latter two were called supplementary evaluations. For this purpose, the vials were opened every five days for rebalancing with normal atmosphere and closed again for incubation to continue.

Embryos from different collection regions were subjected to different drying levels and different incubation temperatures. After the control sample (no drying) was removed, the embryos were subjected to two levels of intermittent drying in a forced air circulation oven, in simple layers without overlapping. The levels were alternated: ten hours at $30 \pm 1{ }^{\circ} \mathrm{C}$ and fourteen hours at $22 \pm 2{ }^{\circ} \mathrm{C}$ (Bonjovani and Barbedo, 2008), until their water contents were close to $53 \%$ (level 1 ) and $46 \%$ (level 2), which are reference values for sensitivity analysis to desiccation of embryos of Inga vera (Bonjovani and Barbedo, 2008; Lamarca and Barbedo, 2015).

Thus, three levels of drying were achieved. At the end of each drying period, the water content and water potential of the embryos were determined again, as described above. Then, ninety embryos (three replicates of thirty embryos each) of each drying level were incubated for fifteen days in respirometers (described above) at $-4{ }^{\circ} \mathrm{C},-2{ }^{\circ} \mathrm{C}, 2{ }^{\circ} \mathrm{C}$ and $5{ }^{\circ} \mathrm{C}$, in the absence of light, for assessments of respiratory rates. Respiratory rates were assessed at five, ten and fifteen days of incubation, as described above. At the end of the last evaluation (fifteen days), samples of the incubated embryos were evaluated for germination and electrical conductivity, as described above.

The experimental design was completely randomized, in a $3 \times 4$ factorial scheme (drying levels $\mathrm{x}$ incubation temperatures), with three replications. Analyses were performed separately for each collection region and the data underwent analysis of variance ( $\mathrm{F}$ test) at $5 \%$. When pertinent, the means were compared with each other by Tukey's test, also at 5\% (Santana and Ranal 2004). Simple correlation coefficients were also calculated for all sampling regions and drying levels across data on water content, water potential (in module), germination, development of normal seedlings and electrical conductivity at incubation temperatures of $-4{ }^{\circ} \mathrm{C},-2{ }^{\circ} \mathrm{C}, 2^{\circ} \mathrm{C}$ and $5{ }^{\circ} \mathrm{C}$, with the significance of $r$ values determined by the t-test at $5 \%$ (Santana and Ranal 2004). 


\section{RESULTS AND DISCUSSION}

The period between the maximum flowering and the collection of ripe fruits of the $I$. vera embryos in the different regions ranged from 96 to 115 days, and the cumulative degrees-day, from 1,424 to $1,739{ }^{\circ} \mathrm{C}$ day. In both of them, there was a variation of less than $20 \%$, despite a large variation in the rainfall regime, from 591 to $1,361 \mathrm{~mm}$ (Table 1). The values of water content (close to 60\%), dry weight (ca. 0.3 g.embryo ${ }^{-1}$ ), water potential (ca. $-1 \mathrm{MPa}$ ), germination, development of normal seedlings (close to 100\%) and electrical conductivity (ca. $11 \mu \mathrm{S} . \mathrm{cm}^{-1} \cdot \mathrm{gDW}^{-1}$ ) of embryos from different regions (Table 1) are similar to those commonly found for the species. However, mean time to germination showed an important difference, especially for RIB embryos that germinated more slowly (Table 1). The consumption values of $\mathrm{O}_{2}$, consumption, $\mathrm{CO}_{2}$ release and $\mathrm{RQ}$ (Table 1 ) suggest a high but balanced respiratory activity, without major differences between $\mathrm{O}_{2}$ and $\mathrm{CO}_{2}$.

The water content of the embryos after drying was similar to the target values (Table 2), especially after the first drying (drying level 1). The initial values (Table 1 ) were actually very similar to those found at the time of setting up the experiments (Table 2). This result indicates negligible variation during the period between harvesting and the beginning of the experiments. Water potential, in turn, was also reduced, as with water content, but not homogeneously (Table 2).

Embryos that were not dried and those from all collection regions did not germinate after fifteen days of incubation at $-4{ }^{\circ} \mathrm{C}$, but all of them germinated at $-2{ }^{\circ} \mathrm{C}$ (Table 3). Drying to level 1 allowed some embryos from RIB and PIR to survive at $-4{ }^{\circ} \mathrm{C}$, but not those from SAP and PAR. On the other hand, drying up to level 2 promoted greater freezing tolerance, increasing the percentage of RIB and PIR embryos that not only survived, but also produced normal seedlings, especially those from PIR (Table 3).

The respiratory rates of the embryos decreased as the incubation temperature decreased for embryos from all collection regions, a fact that did not occur for their drying levels (Table 4). In some situations, RQ was close to 1 , while in others, there was an increase or decrease (Table 5), ranging from 0.52 (probable non-respiratory oxidation) to 2.84 (probable anaerobic respiration), indicating a change in the respiratory pattern, with changes in both temperature and drying level.

Table 1. Collection regions and initial characterization of newly collected Inga vera embryos.

\begin{tabular}{|c|c|c|c|c|}
\hline \multirow{2}{*}{$\begin{array}{ll} & \text { Characteristics of } \\
\text { Region } & \end{array}$} & \multicolumn{4}{|c|}{ embryo-collecting } \\
\hline & RIB & PIR & SAP & PAR \\
\hline Fruit ripening cycle (days) & 107 & 96 & 115 & 106 \\
\hline Degree-day ( ${ }^{\circ} \mathrm{C}$ day) & 1,485 & 1,424 & 1,605 & 1,739 \\
\hline Rainfall (mm) & 652 & 591 & 1,361 & 896 \\
\hline Water content (\%) & $58.4 \pm 0.1$ & $62.3 \pm 1.3$ & $56.3 \pm 1.5$ & $61.2 \pm 1.9$ \\
\hline Water potential (MPa) & $-1.38 \pm 0.08$ & $-1.30 \pm 0.21$ & $-0.82 \pm 0.14$ & $-0.91 \pm 0.09$ \\
\hline Dry weight (g.embryo ${ }^{-1}$ ) & $0.44 \pm 0.02$ & $0.33 \pm 0.06$ & $0.26 \pm 0.02$ & $0.25 \pm 0.03$ \\
\hline Germination (\%) & $100 \pm 0$ & $100 \pm 0$ & $100 \pm 0$ & $98 \pm 3$ \\
\hline Normal seedlings (\%) & $90 \pm 5$ & $98 \pm 3$ & $100 \pm 0$ & $98 \pm 3$ \\
\hline Mean time (days) & $6.15 \pm 0.15$ & $3.90 \pm 0.30$ & $3.45 \pm 0.15$ & $3.86 \pm 0.21$ \\
\hline Electrical conductivity $\left(\mu \mathrm{S} \cdot \mathrm{cm}^{-1} \cdot \mathrm{gDW}^{-1}\right)$ & $11.77 \pm 2.74$ & $11.71 \pm 0.49$ & $11.18 \pm 2.23$ & $11.56 \pm 1.88$ \\
\hline $\mathrm{O}_{2}$ consumption $\left(\mu \mathrm{mol} \mathrm{O}_{2} \cdot g \mathrm{gW}^{-1} \cdot \mathrm{d}^{-1}\right)$ & $123.97 \pm 11.45$ & $167.48 \pm 19.26$ & $150.96 \pm 19.66$ & $161.83 \pm 12.55$ \\
\hline $\mathrm{CO}_{2}$ release $\left(\mu \mathrm{mol} \mathrm{CO}{ }_{2} \cdot \mathrm{gDW}^{-1} \cdot \mathrm{d}^{-1}\right)$ & $98.14 \pm 2.33$ & $149.29 \pm 13.15$ & $133.91 \pm 18.65$ & $132.47 \pm 10.05$ \\
\hline Respiratory quotient $\left(\mathrm{CO}_{2} \cdot \mathrm{O}_{2}{ }^{-1}\right)$ & $0.80 \pm 0.07$ & $0.89 \pm 0.02$ & $0.89 \pm 0.01$ & $0.82 \pm 0.01$ \\
\hline
\end{tabular}

RIB: Ribeirão Vermelho; PIR: Piracicaba; SAP: São Paulo; PAR: Pariquera-Açu. Mean values accompanied by standard deviation. 
Table 2. Values found for water content and water potential of Inga vera embryos before (no drying) and after two levels of drying.

\begin{tabular}{cccc}
\hline Collection region & Drying level & Water content (\%) & Water potential (MPa) \\
\hline \multirow{3}{*}{ Ribeirão Vermelho } & No drying & $58.6 \pm 0.9$ & $-1.10 \pm 0.08$ \\
& Level 1 & $53.4 \pm 2.3$ & $-2.69 \pm 0.44$ \\
& Level 2 & $43.8 \pm 1.1$ & $-4.71 \pm 0.43$ \\
\hline \multirow{2}{*}{ Piracicaba } & No drying & $63.4 \pm 1.1$ & $-1.15 \pm 0.17$ \\
& Level 1 & $53.0 \pm 2.2$ & $-2.99 \pm 0.31$ \\
São Paulo & Level 2 & $47.6 \pm 0.9$ & $-4.69 \pm 0.19$ \\
& Lo drying & $56.1 \pm 1.9$ & $-0.77 \pm 0.24$ \\
& Level 1 & $54.1 \pm 4.1$ & $-2.41 \pm 0.05$ \\
& Level 2 & $50.3 \pm 1.0$ & $-3.05 \pm 0.40$ \\
\hline \multirow{2}{*}{ Pariquera-Açu } & No drying & $59.2 \pm 1.6$ & $-0.89 \pm 0.15$ \\
& Level 1 & $55.3 \pm 1.1$ & $-2.33 \pm 0.05$ \\
& Level 2 & $49.4 \pm 2.9$ & $-3.50 \pm 0.47$ \\
\hline
\end{tabular}

Mean values accompanied by standard deviation.

Electrical conductivity (EC) was higher in embryos that were not dried, regardless of the collection region, after fifteen days of incubation at $-4{ }^{\circ} \mathrm{C}$. As both incubation temperature $\left(-2{ }^{\circ} \mathrm{C}, 2{ }^{\circ} \mathrm{C}\right.$ and $5{ }^{\circ} \mathrm{C}$ ) and drying levels (levels 1 and 2 ) were increased, EC values decreased (Table 5). The results for EC showed the embryos whose cell membrane system integrity was ruptured or preserved with drying and freezing at $-4{ }^{\circ} \mathrm{C}$. For the embryos from RIB and PIR, it is evident that drying levels 1 and 2, especially the latter, favored the preservation of membrane system integrity, something that did not occur for the embryos from SAP and PAR (Table 5). At the other incubation temperatures at $-2{ }^{\circ} \mathrm{C}, 2^{\circ} \mathrm{C}$ and $5{ }^{\circ} \mathrm{C}$, the increase in temperature corresponded to the preservation of cell membrane system integrity for all regions of collection. Furthermore, the EC results were similar to those of germination and development of normal seedlings (Tables 3 and 5).

The results of the initial characterization showed that, although all the fruits had been collected when they were ripe, their embryos showed maturation differences: those of RIB, for example, were closer to physiological maturity than the others, because: a) although the embryos from all regions had very high water content, above $50 \%$, typical for species of the genus Inga (Mata et al., 2013; Schulz et al., 2014), those from RIB had the lowest water potential. This result indicates more binding sites, therefore a better structured matrix (Vertucci and Farrant, 1995); b) the embryos accumulated more dry weight (Marcos-Filho, 2015); c) they had a lower respiration rate, indicating that they were among more mature embryos (the more mature, the lower the respiratory rate, Araujo and Barbedo, 2017) and embryos that had already started germination (the more advanced in germination, the higher the respiratory rates, Hell et al., 2019); d) they had the longest mean germination time, indicating that they had not started germination before harvest, which is relatively common for embryos of this species (Andreo et al., 2006; Bonjovani and Barbedo, 2020).

Embryos from other regions were collected during their germination process, as average germination time for all of them is much shorter than those of RIB, and could not be explained by greater vigor, because as stated above, RIB embryos would be the most advanced in the process of maturation and, therefore, the most vigorous. The SAP embryos had been harvested at an advanced stage of maturation, but they were not mature enough, because although they were released from the plant with the lowest water content, they had little dry weight accumulation and little formation of the cellular matrix structure, because the water potential indicates the presence of a great deal of free water, even with a lower water content. 
Table 3. Germination and development of normal seedlings from Inga vera embryos with different drying levels and submitted to different incubation temperatures for fifteen days.

\begin{tabular}{|c|c|c|c|c|c|c|}
\hline \multirow{2}{*}{$\begin{array}{l}\text { Region of } \\
\text { collection }\end{array}$} & \multirow{2}{*}{ Level of drying } & \multicolumn{4}{|c|}{ Temperature $\left({ }^{\circ} \mathrm{C}\right)$} & \multirow{2}{*}{ Means } \\
\hline & & -4 & -2 & 2 & 5 & \\
\hline & \multicolumn{6}{|c|}{ Germination (\%) } \\
\hline \multirow{4}{*}{ RIB } & No drying & $0 \mathrm{cC}$ & $100 \mathrm{aA}$ & $97 \mathrm{aB}$ & $100 \mathrm{aA}$ & - \\
\hline & Level 1 & $30 \mathrm{bc}$ & $100 \mathrm{aA}$ & $97 \mathrm{aB}$ & $100 \mathrm{aA}$ & - \\
\hline & Level 2 & $83 \mathrm{aB}$ & $83 \mathrm{bB}$ & $87 \mathrm{bA}$ & $70 \mathrm{bc}$ & - \\
\hline & \multicolumn{2}{|c|}{ Coefficient of variation } & $15.38 \%$ & & & \\
\hline \multirow{4}{*}{ PIR } & No drying & $0 \mathrm{cC}$ & $93 \mathrm{aB}$ & $100 \mathrm{aA}$ & $100 \mathrm{aA}$ & - \\
\hline & Level 1 & $30 \mathrm{bc}$ & $93 \mathrm{aB}$ & $100 \mathrm{aA}$ & $100 \mathrm{aA}$ & - \\
\hline & Level 2 & $87 \mathrm{aD}$ & $93 \mathrm{aB}$ & $90 \mathrm{bC}$ & $100 \mathrm{aA}$ & - \\
\hline & \multicolumn{2}{|c|}{ Coefficient of variation } & $13.75 \%$ & & & \\
\hline \multirow{5}{*}{ SAP } & No drying & 0 & 87 & 97 & 100 & $71 \mathrm{c}$ \\
\hline & Level 1 & 0 & 97 & 100 & 100 & $74 \mathrm{~b}$ \\
\hline & Level 2 & 0 & 100 & 100 & 100 & 75 a \\
\hline & Means & $O D$ & $94 \mathrm{C}$ & $99 \mathrm{~B}$ & $100 \mathrm{~A}$ & \\
\hline & \multicolumn{2}{|c|}{ Coefficient of variation } & $5.57 \%$ & & & \\
\hline \multirow{5}{*}{ PAR } & No drying & $0 \mathrm{aD}$ & $90 \mathrm{cB}$ & $83 c C$ & $100 \mathrm{aA}$ & - \\
\hline & Level 1 & $0 \mathrm{aB}$ & $100 \mathrm{aA}$ & $100 \mathrm{aA}$ & $100 \mathrm{aA}$ & - \\
\hline & Level 2 & $0 \mathrm{aC}$ & $93 \mathrm{bB}$ & $97 \mathrm{bA}$ & $97 \mathrm{bA}$ & - \\
\hline & \multicolumn{2}{|c|}{ Coefficient of variation } & \multicolumn{2}{|l|}{$6.15 \%$} & & \\
\hline & \multicolumn{5}{|c|}{ Normal seedlings (\%) } & \\
\hline \multirow{4}{*}{ RIB } & No drying & $0 \mathrm{cD}$ & $87 \mathrm{bC}$ & $93 \mathrm{aB}$ & 97 aA & - \\
\hline & Level 1 & $13 \mathrm{bD}$ & $100 \mathrm{aA}$ & $93 \mathrm{aC}$ & $97 \mathrm{aB}$ & - \\
\hline & Level 2 & $53 \mathrm{aC}$ & $53 \mathrm{cC}$ & $73 \mathrm{bA}$ & $60 \mathrm{bB}$ & - \\
\hline & \multicolumn{2}{|c|}{ Coefficient of variation } & $13.36 \%$ & & & \\
\hline \multirow{4}{*}{ PIR } & No drying & $0 \mathrm{cD}$ & $90 \mathrm{bC}$ & $97 \mathrm{aB}$ & $100 \mathrm{aA}$ & - \\
\hline & Level 1 & $10 \mathrm{bC}$ & $93 \mathrm{aB}$ & $97 \mathrm{aA}$ & $97 \mathrm{bA}$ & - \\
\hline & Level 2 & $77 \mathrm{aD}$ & $87 \mathrm{cC}$ & $90 \mathrm{bB}$ & $97 \mathrm{bA}$ & - \\
\hline & \multicolumn{2}{|c|}{ Coefficient of variation } & $11.34 \%$ & & & \\
\hline \multirow{5}{*}{ SAP } & No drying & 0 & 87 & 97 & 100 & $71 \mathrm{~b}$ \\
\hline & Level 1 & 0 & 93 & 100 & 97 & $73 a$ \\
\hline & Level 2 & 0 & 93 & 90 & 97 & $70 \mathrm{c}$ \\
\hline & Means & $O D$ & $91 \mathrm{C}$ & $96 \mathrm{~B}$ & $98 \mathrm{~A}$ & \\
\hline & \multicolumn{2}{|c|}{ Coefficient of variation } & $7.03 \%$ & & & \\
\hline \multirow{5}{*}{ PAR } & No drying & 0 & 83 & 80 & 97 & $65 c$ \\
\hline & Level 1 & 0 & 93 & 97 & 100 & $73 \mathrm{a}$ \\
\hline & Level 2 & 0 & 90 & 97 & 93 & $70 \mathrm{~b}$ \\
\hline & Means & OD & $89 \mathrm{C}$ & $91 \mathrm{~B}$ & $97 \mathrm{~A}$ & \\
\hline & \multicolumn{2}{|c|}{ Coefficient of variation } & $9.33 \%$ & & & \\
\hline
\end{tabular}

RIB: Ribeirão Vermelho; PIR: Piracicaba; SAP: São Paulo; PAR: Pariquera-Açu.

Means followed by the same letter (lowercase in columns, uppercase in rows) do not differ (Tukey, 5\%). 
Table 4. Oxygen $\left(\mathrm{O}_{2}\right)$ consumption and production of carbon dioxide $\left(\mathrm{CO}_{2}\right)$ of Inga vera embryos at different drying levels and incubated for five days at different temperatures.

\begin{tabular}{|c|c|c|c|c|c|c|}
\hline \multirow{2}{*}{$\begin{array}{l}\text { Region of } \\
\text { collection }\end{array}$} & \multirow{2}{*}{ Level of drying } & \multicolumn{4}{|c|}{ Temperature $\left({ }^{\circ} \mathrm{C}\right)$} & \multirow{2}{*}{ Means } \\
\hline & & -4 & -2 & 2 & 5 & \\
\hline & \multicolumn{6}{|c|}{$\mathrm{O}_{2}$ consumption $\left(\mu \mathrm{mol} \mathrm{O}_{2} \cdot g \mathrm{gW}^{-1} \cdot \mathrm{d}^{-1}\right)$} \\
\hline \multirow{5}{*}{ RIB } & No drying & 13.59 & 20.93 & 16.25 & 22.52 & $18.32 \mathrm{a}$ \\
\hline & Level 1 & 12.93 & 9.43 & 19.65 & 22.80 & $16.20 \mathrm{~b}$ \\
\hline & Level 2 & 11.54 & 9.84 & 17.06 & 20.79 & $14.81 \mathrm{c}$ \\
\hline & Means & $12.69 \mathrm{D}$ & $13.40 \mathrm{C}$ & $17.65 \mathrm{~B}$ & $22.04 \mathrm{~A}$ & \\
\hline & \multicolumn{2}{|c|}{ Coefficient of variation } & $37.66 \%$ & & & \\
\hline \multirow{5}{*}{ PIR } & No drying & 22.63 & 17.29 & 29.41 & 54.43 & $30.94 \mathrm{a}$ \\
\hline & Level 1 & 23.11 & 12.12 & 29.55 & 34.80 & $24.90 \mathrm{~b}$ \\
\hline & Level 2 & 7.07 & 7.03 & 11.03 & 15.86 & $10.25 \mathrm{c}$ \\
\hline & Means & $17.61 \mathrm{C}$ & $12.14 \mathrm{D}$ & $23.33 \mathrm{~B}$ & $35.03 \mathrm{~A}$ & \\
\hline & \multicolumn{2}{|c|}{ Coefficient of variation } & $74.13 \%$ & & & \\
\hline \multirow{4}{*}{ SAP } & No drying & $4.54 \mathrm{cD}$ & $7.25 \mathrm{cB}$ & $7.14 \mathrm{cC}$ & $22.79 \mathrm{aA}$ & - \\
\hline & Level 1 & $5.66 \mathrm{bD}$ & $9.44 \mathrm{aC}$ & $16.83 \mathrm{bA}$ & $16.79 c B$ & - \\
\hline & Level 2 & $7.96 \mathrm{aD}$ & $8.45 \mathrm{bC}$ & $19.66 \mathrm{aB}$ & $19.87 \mathrm{bA}$ & - \\
\hline & \multicolumn{2}{|c|}{ Coefficient of variation } & $32.86 \%$ & & & \\
\hline \multirow{6}{*}{ PAR } & No drying & 21.43 & 20.42 & 34.49 & 36.58 & $28.23 b$ \\
\hline & Level 1 & 14.63 & 18.94 & 33.64 & 32.14 & $24.84 \mathrm{c}$ \\
\hline & Level 2 & 20.56 & 23.82 & 31.90 & 40.27 & $29.14 \mathrm{a}$ \\
\hline & Means & $18.88 \mathrm{D}$ & $21.06 \mathrm{C}$ & $33.35 \mathrm{~B}$ & $36.33 \mathrm{~A}$ & \\
\hline & \multicolumn{2}{|c|}{ Coefficient of variation } & $23.14 \%$ & & & \\
\hline & \multicolumn{5}{|c|}{$\mathrm{CO}_{2}$ production $\left(\mu \mathrm{mol} \mathrm{CO}_{2} \cdot \mathrm{gDW}^{-1} \cdot \mathrm{d}^{-1}\right)$} & \\
\hline \multirow{5}{*}{$\mathrm{RIB}$} & No drying & 14.10 & 7.56 & 18.13 & 23.06 & $15.71 \mathrm{c}$ \\
\hline & Level 1 & 14.38 & 13.14 & 21.35 & 23.47 & $18.09 \mathrm{a}$ \\
\hline & Level 2 & 14.97 & 12.66 & 18.44 & 21.04 & $16.78 \mathrm{~b}$ \\
\hline & Means & $14.48 \mathrm{C}$ & $11.12 \mathrm{D}$ & $19.30 \mathrm{~B}$ & $22.52 \mathrm{~A}$ & \\
\hline & \multicolumn{2}{|c|}{ Coefficient of variation } & $29.92 \%$ & & & \\
\hline \multirow{5}{*}{ PIR } & No drying & 17.85 & 17.93 & 27.21 & 32.19 & $23.80 \mathrm{~b}$ \\
\hline & Level 1 & 23.10 & 14.25 & 28.48 & 32.39 & $24.56 \mathrm{a}$ \\
\hline & Level 2 & 12.30 & 15.54 & 13.80 & 16.90 & $14.64 \mathrm{c}$ \\
\hline & Means & $17.75 \mathrm{C}$ & $15.91 \mathrm{D}$ & $23.17 \mathrm{~B}$ & $27.16 \mathrm{~A}$ & \\
\hline & \multicolumn{2}{|c|}{ Coefficient of variation } & $36.97 \%$ & & & \\
\hline \multirow{4}{*}{ SAP } & No drying & $12.59 \mathrm{cD}$ & $14.11 \mathrm{cB}$ & $13.83 \mathrm{cC}$ & $26.82 \mathrm{aA}$ & - \\
\hline & Level 1 & 14.86 bD & $16.79 \mathrm{bB}$ & $15.65 \mathrm{bC}$ & $22.61 \mathrm{cA}$ & - \\
\hline & Level 2 & $15.24 \mathrm{aD}$ & $17.16 \mathrm{aC}$ & $26.30 \mathrm{aB}$ & $26.52 \mathrm{bA}$ & - \\
\hline & \multicolumn{2}{|c|}{ Coefficient of variation } & $15.30 \%$ & & & \\
\hline \multirow{5}{*}{ PAR } & No drying & 18.15 & 13.25 & 23.13 & 23.76 & $19.57 \mathrm{~b}$ \\
\hline & Level 1 & 12.75 & 12.44 & 23.97 & 18.51 & $16.91 \mathrm{c}$ \\
\hline & Level 2 & 16.44 & 15.95 & 23.71 & 28.85 & $21.24 \mathrm{a}$ \\
\hline & Means & $15.78 \mathrm{C}$ & $13.88 \mathrm{D}$ & $23.60 \mathrm{~B}$ & $23.71 \mathrm{~A}$ & \\
\hline & \multicolumn{2}{|c|}{ Coefficient of variation } & $34.97 \%$ & & & \\
\hline
\end{tabular}

RIB: Ribeirão Vermelho; PIR: Piracicaba; SAP: São Paulo; PAR: Pariquera-Açu.

Means followed by the same letter (lowercase in columns, uppercase in rows) do not differ (Tukey, 5\%). 
Table 5. Electrical conductivity and respiratory quotient (RQ) of Inga vera embryos with different drying levels and submitted at different incubation temperatures for fifteen days.

\begin{tabular}{|c|c|c|c|c|c|c|}
\hline \multirow{2}{*}{$\begin{array}{l}\text { Region of } \\
\text { collection }\end{array}$} & \multirow{2}{*}{ Level of drying } & \multicolumn{4}{|c|}{ Temperature $\left({ }^{\circ} \mathrm{C}\right)$} & \multirow{2}{*}{ Means } \\
\hline & & -4 & -2 & 2 & 5 & \\
\hline & \multicolumn{6}{|c|}{ Electrical conductivity $\left(\mu \mathrm{S} \cdot \mathrm{cm}^{-1} \cdot \mathrm{gDW}^{-1}\right)$} \\
\hline \multirow{4}{*}{ RIB } & No drying & $393.54 \mathrm{aA}$ & $40.40 \mathrm{bB}$ & $27.94 \mathrm{bC}$ & $21.00 \mathrm{cD}$ & - \\
\hline & Level 1 & $284.50 \mathrm{bA}$ & $36.28 \mathrm{cB}$ & 13.99 cD & $22.49 \mathrm{bC}$ & - \\
\hline & Level 2 & $92.03 \mathrm{cA}$ & $69.02 \mathrm{aB}$ & $50.74 \mathrm{aC}$ & $49.08 \mathrm{aD}$ & - \\
\hline & \multicolumn{2}{|c|}{ Coefficient of variation } & $33.16 \%$ & & & \\
\hline \multirow{4}{*}{ PIR } & No drying & $401.35 \mathrm{aA}$ & $38.80 \mathrm{bB}$ & $27.23 \mathrm{bC}$ & 18.44 cD & - \\
\hline & Level 1 & 254.27 bA & $37.53 \mathrm{cC}$ & 21.37 cD & $39.82 \mathrm{bB}$ & - \\
\hline & Level 2 & $70.22 \mathrm{cA}$ & $57.27 \mathrm{aB}$ & $34.84 \mathrm{aD}$ & $46.29 \mathrm{aC}$ & - \\
\hline & \multicolumn{2}{|c|}{ Coefficient of variation } & $39.39 \%$ & & & \\
\hline \multirow{5}{*}{ SAP } & No drying & 389.04 & 33.34 & 12.45 & 18.04 & $113.22 \mathrm{c}$ \\
\hline & Level 1 & 401.47 & 39.62 & 19.79 & 20.27 & $120.29 \mathrm{~b}$ \\
\hline & Level 2 & 399.35 & 53.23 & 41.19 & 27.68 & $130.36 \mathrm{a}$ \\
\hline & Means & $396.62 \mathrm{~A}$ & $42.06 \mathrm{~B}$ & $24.48 \mathrm{C}$ & $22.00 \mathrm{D}$ & - \\
\hline & \multicolumn{2}{|c|}{ Coefficient of variation } & $17.13 \%$ & & & \\
\hline \multirow{6}{*}{ PAR } & No drying & 440.32 & 37.34 & 88.31 & 50.23 & $154.05 a$ \\
\hline & Level 1 & 364.24 & 35.84 & 35.47 & 36.92 & 118.12 c \\
\hline & Level 2 & 419.89 & 62.04 & 58.33 & 73.97 & $153.56 \mathrm{~b}$ \\
\hline & Means & $408.15 \mathrm{~A}$ & $45.08 \mathrm{D}$ & $60.70 \mathrm{~B}$ & $53.71 \mathrm{C}$ & - \\
\hline & \multicolumn{2}{|c|}{ Coefficient of variation } & \multicolumn{2}{|l|}{$19.97 \%$} & & \\
\hline & \multicolumn{5}{|c|}{ Respiratory quotient $\left(\mathrm{CO}_{2} \cdot \mathrm{O}_{2}^{-1}\right)$} & \\
\hline \multirow{4}{*}{ RIB } & No drying & $1.00 \mathrm{cC}$ & $0.72 \mathrm{cD}$ & $1.18 \mathrm{aA}$ & $1.04 \mathrm{aB}$ & - \\
\hline & Level 1 & $1.20 \mathrm{bB}$ & $1.41 \mathrm{aA}$ & $1.09 \mathrm{bC}$ & $1.03 \mathrm{bD}$ & - \\
\hline & Level 2 & $1.28 \mathrm{aB}$ & $1.29 \mathrm{bA}$ & $1.08 \mathrm{cC}$ & $1.01 \mathrm{cD}$ & - \\
\hline & \multicolumn{2}{|c|}{ Coefficient of variation } & $18.68 \%$ & & & \\
\hline \multirow{4}{*}{ PIR } & No drying & $0.75 \mathrm{cC}$ & $0.63 \mathrm{cD}$ & $0.93 \mathrm{cA}$ & $0.79 \mathrm{bB}$ & - \\
\hline & Level 1 & $1.00 \mathrm{bB}$ & $1.39 \mathrm{bA}$ & $0.99 \mathrm{bC}$ & $0.94 \mathrm{aD}$ & - \\
\hline & Level 2 & $1.75 \mathrm{aB}$ & $2.25 \mathrm{aA}$ & $1.41 \mathrm{aC}$ & $0.70 \mathrm{cD}$ & - \\
\hline & \multicolumn{2}{|c|}{ Coefficient of variation } & $31.02 \%$ & & & \\
\hline \multirow{5}{*}{ SAP } & No drying & 2.84 & 2.03 & 1.06 & 1.18 & $1.78 \mathrm{a}$ \\
\hline & Level 1 & 2.72 & 1.78 & 1.09 & 1.47 & $1.77 \mathrm{~b}$ \\
\hline & Level 2 & 2.20 & 2.08 & 1.34 & 1.34 & $1.74 \mathrm{c}$ \\
\hline & Means & $2.59 \mathrm{~A}$ & $1.96 \mathrm{~B}$ & $1.16 \mathrm{D}$ & $1.33 \mathrm{C}$ & - \\
\hline & \multicolumn{2}{|c|}{ Coefficient of variation } & $28.48 \%$ & & & \\
\hline \multirow{5}{*}{ PAR } & No drying & 0.84 & 0.57 & 0.67 & 0.65 & $0.68 \mathrm{~b}$ \\
\hline & Level 1 & 0.71 & 0.65 & 0.71 & 0.52 & $0.65 c$ \\
\hline & Level 2 & 0.80 & 0.60 & 0.74 & 0.72 & $0.72 \mathrm{a}$ \\
\hline & Means & $0.78 \mathrm{~A}$ & $0.61 \mathrm{D}$ & $0.71 \mathrm{~B}$ & $0.63 \mathrm{C}$ & - \\
\hline & \multicolumn{2}{|c|}{ Coefficient of variation } & $32.81 \%$ & & & \\
\hline
\end{tabular}

RIB: Ribeirão Vermelho; PIR: Piracicaba; SAP: São Paulo; PAR: Pariquera-Açu.

Means followed by the same letter (lowercase in columns, uppercase in rows) do not differ (Tukey, 5\%). 
The PAR embryos were very immature embryos since their high values of water content and water potential indicate a great deal of free water, in addition to little dry weight accumulation. And finally, the PIR embryos were in an advanced stage of maturation, but their germination process had already started before harvest, as they showed high dry weight accumulation and little free water, indicating well-formed embryos, which is typical of the most advanced stage of maturation.

However, the shorter average time indicates that germination had already started. Therefore, these (PIR) are embryos at a more advanced stage of maturation than those of SAP and PAR and are very similar to those of RIB. Thus, it was clear that environmental conditions had an important effect on the maturation process of Inga vera seeds until the moment of dispersion, as reported in previous works, also for other species of the same genus (Bonjovani and Barbedo, 2020; Mata et al., 2013).

Drying up to level 1 resulted in embryos whose water content values were close to 54\%, ranging from 53\% to 55\% among the embryos in each region. This way, the water content values of the embryos were very similar. These water contents allowed more reliable comparisons between embryos from different regions. On the other hand, drying up to level 2 resulted in embryos with very different water content values, ranging from $43 \%$ to $50 \%$ among embryos in each region. This result did not allow for a better analysis of the effects of environmental conditions on the formation of these embryos. This range in water content values may be associated with more irregular removal of water from different embryos at this level of hydration. Thus, when level 1 drying was used, the water potentials did not follow a pattern: they were higher for SAP and PAR, indicating a higher proportion of free water. This is of crucial importance, not only because it confirms information about the initial characteristics of different materials, as mentioned above, but also allows an important analysis of the death of embryos when frozen. For example: if we use the water potential of $-3 \mathrm{MPa}$ as a reference, the embryos from PIR would have $53 \%$ water, while those from SAP would have $50 \%$. This means that SAP needed further water content reduction to reach the same potential as PIR.

The results also made it evident that there is a need to remove all water from types 4 and 5 (Vertucci and Farrant, 1995) to enable tolerance to freezing. Although desiccation-sensitive seeds are often considered to be intolerant to freezing (Goldfarb et al., 2017, and references contained), those of Inga vera tolerated sub-zero temperatures, a fact also reported by Bonjovani and Barbedo (2008) for $-2^{\circ} \mathrm{C}$. Seeds stored with high water contents are susceptible to damage caused by freezing temperatures, owing to the formation of ice crystals in the tissues, which leads to loss of viability (Fonseca and Freire, 2003).

In the present study, however, embryos from two populations (RIB and PIR) tolerated up to $-4{ }^{\circ} \mathrm{C}$, while those from two other populations (SAP and PAR) only tolerated up to $-2{ }^{\circ} \mathrm{C}$. These variations may be due to maturation stage, as mentioned by Barbedo (2018). These variations also justify the results of electrical conductivity, which essentially indicate the loss of membrane selectivity and of germination, since seeds evidently lose their germination capacity after cell disruption. They also bring great and important consequences for the development of technology for sub-zero conservation, as they represent a narrow range of water content in which these embryos can be dried (without losing viability because of their sensitivity to desiccation), but remain able to tolerate some freezing. Also, the origin and maturation stage of the embryos should be considered carefully. Notably, only embryos from more advanced stages of maturation, from two sources, tolerated the lowest temperatures. This is certainly due both to the greater ability to tolerate the removal of type 4 and 5 waters (Vertucci and Farrant, 1995), and to a better cytoplasmic structure (Walters, 2015) - both of which reduce the effects of water crystallization inside the cells. In this regard, electrical conductivity is an important tool to identify freezing-induced membrane rupture.

The analysis of respiratory rates clearly showed that the change in gas composition definitely modifies the respiration pattern of the embryos (Figure 1). Therefore, when I. vera embryos need to be stored, airtight packaging should be used, as the embryo's own respiration will make the environment harmful. Importantly, this happens even at freezing temperatures, at which embryos continue to respire. This was evidenced by the results of $\mathrm{O}_{2}$ consumption and $\mathrm{CO}_{2}$ release at negative temperatures: although respiration of $I$. vera embryos decreases with decreasing temperature, it still occurs down to $-4{ }^{\circ} \mathrm{C}$. 

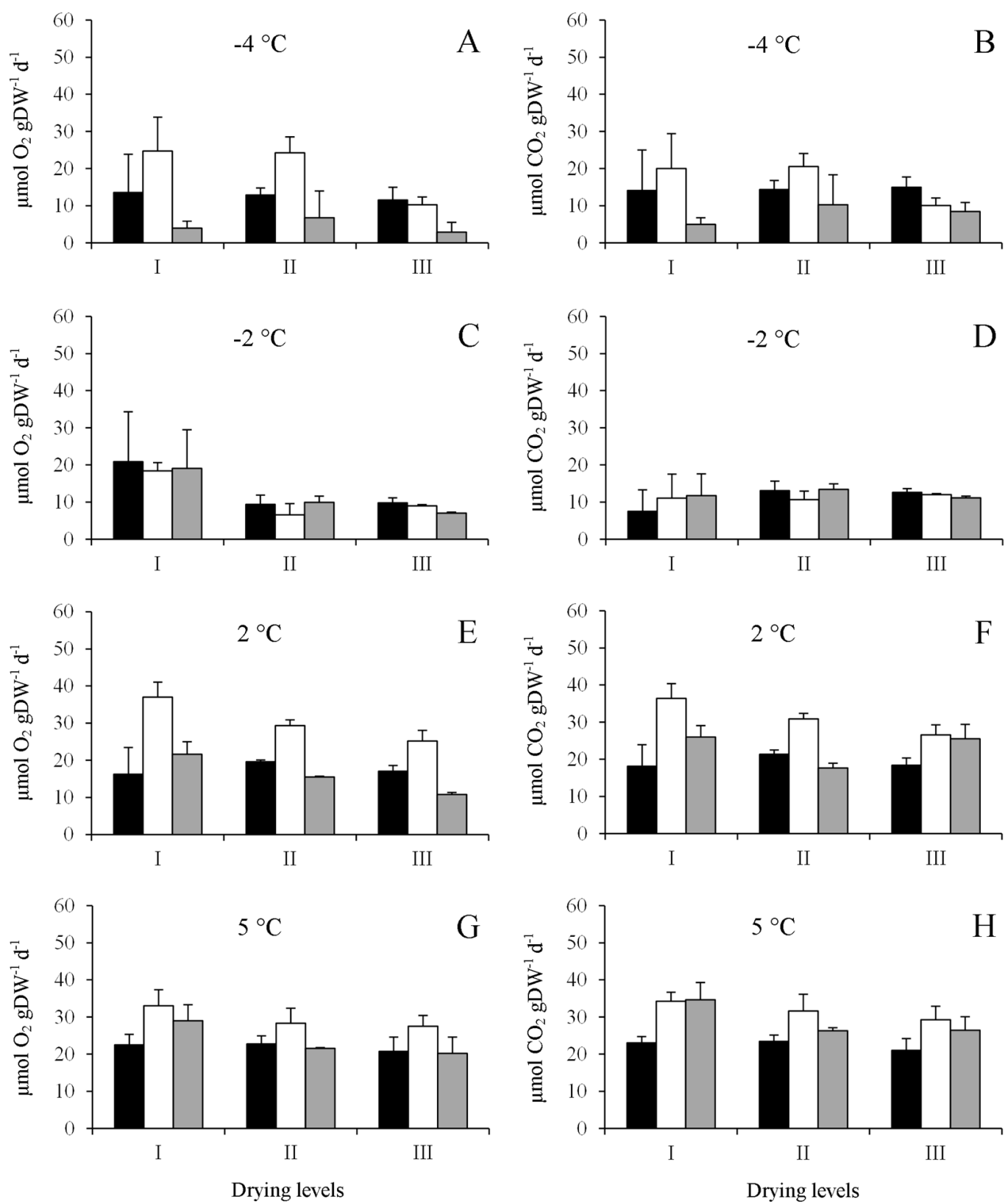

I: no drying; II: level 1 drying; III: level 2 drying. Bars represent mean values accompanied by standard deviation. Black bars: first evaluation; white bars: second evaluation; gray bars: third evaluation.

Figure 1. Values of all evaluations (total of three, that is, at five, ten and fifteen days of incubation) of oxygen $\left(\mathrm{O}_{2}\right)$ consumption and the production of carbon dioxide $\left(\mathrm{CO}_{2}\right)$ of Inga vera embryos from RIB (Ribeirão Vermelho), with different drying levels and submitted to different incubation temperatures. Figures $A, C, E, G$ represent $\mathrm{O}_{2}$ consumption. Figures $\mathrm{B}, \mathrm{D}, \mathrm{F}, \mathrm{H}$ represent $\mathrm{CO}_{2}$ production.

Thus, one cannot consider storing I. vera embryos merely by lowering the temperature and expecting metabolism to stop: they will continue to respire. As shown in previous studies (Araujo and Barbedo, 2017; Bonjovani and Barbedo, 2019; Guardia et al., 2020), there were no patterns of change in respiration with reduction of water content for most embryos from different regions. This result differs from the outcomes for desiccation-tolerant seeds in general, or even for some desiccation-sensitive seeds, such as Castanea sativa (Leprince et al., 1999) and Eugenia pyriformis (Lamarca et al., 2020). 
Table 6. Simple correlation coefficients ( $r$ ) between water content $(W C)$, water potential ( $\Psi$, in module), germination (G), normal seedling development (NS) and electrical conductivity (EC) of Inga vera embryos from different regions, with different drying levels and incubated at $-4{ }^{\circ} \mathrm{C},-2{ }^{\circ} \mathrm{C}, 2^{\circ} \mathrm{C}$ and $5{ }^{\circ} \mathrm{C}$.

\begin{tabular}{|c|c|c|c|c|}
\hline & $|\Psi|$ & $\mathrm{G}$ & NS & EC \\
\hline \multicolumn{5}{|c|}{ Embryos incubated at $-4^{\circ} \mathrm{C}$} \\
\hline WC & $-0.92^{*}$ & $-0.70^{*}$ & $-0.67^{*}$ & $0.69^{*}$ \\
\hline$|\Psi|$ & & $0.79^{*}$ & $0.76^{*}$ & $-0.78^{*}$ \\
\hline G & & & $0.97^{*}$ & $-0.99^{*}$ \\
\hline \multirow[t]{2}{*}{ NS } & & & & $-0.95^{*}$ \\
\hline & \multicolumn{4}{|c|}{ Embryos incubated at $-2^{\circ} \mathrm{C}$} \\
\hline WC & $-0.92^{*}$ & $0.34^{\mathrm{NS}}$ & $0.49^{\mathrm{NS}}$ & $-0.76^{*}$ \\
\hline$|\Psi|$ & & $-0.19^{N S}$ & $-0.34^{\text {NS }}$ & $0.73^{*}$ \\
\hline G & & & $0.76^{*}$ & $-0.38^{\mathrm{NS}}$ \\
\hline \multirow[t]{2}{*}{ NS } & & & & $-0.56^{\mathrm{NS}}$ \\
\hline & \multicolumn{4}{|c|}{ Embryos incubated at $2^{\circ} \mathrm{C}$} \\
\hline WC & $-0.92^{*}$ & $0.27^{\mathrm{NS}}$ & $0.42^{\mathrm{NS}}$ & $-0.16^{\mathrm{NS}}$ \\
\hline$|\Psi|$ & & $-0.19^{\mathrm{NS}}$ & $-0.32^{\mathrm{NS}}$ & $0.08^{\mathrm{NS}}$ \\
\hline G & & & $0.86^{*}$ & $-0.70^{*}$ \\
\hline \multirow[t]{2}{*}{ NS } & & & & $-0.64^{*}$ \\
\hline & & \multicolumn{3}{|c|}{ Embryos incubated at $5^{\circ} \mathrm{C}$} \\
\hline WC & $-0.92^{*}$ & $0.60^{*}$ & $0.65^{*}$ & $-0.52^{\mathrm{NS}}$ \\
\hline$|\Psi|$ & & $-0.53^{\mathrm{NS}}$ & $-0.57^{*}$ & $0.53^{\mathrm{NS}}$ \\
\hline G & & & $0.99^{*}$ & $-0.33^{\mathrm{NS}}$ \\
\hline NS & & & & $-0.36^{\mathrm{NS}}$ \\
\hline
\end{tabular}

$(*)$ : significant $r$ at $5 \%$ probability; (NS): non-significant $r$.

However, there are very few studies on respiratory rates of desiccation-tolerant seeds in their immature phases, when they are still sensitive. According to Barbedo (2018), in these immature phases, such seeds are similar to desiccation-sensitive seeds and, therefore, their respiration patterns could also change when water content is reduced.

Finally, the correlations between the variables (Table 6) showed that the higher the water content, the higher the electrical conductivity and the lower the germination and seedling development values for normal seedlings at freezing temperatures, especially at $-4{ }^{\circ} \mathrm{C}$. This relationship also occurs between water potential and those variables (electrical conductivity, germination and development of normal seedlings), but even more evidently, as the values of these correlations are higher than those that relate such variables to water content.

Therefore, although the dry seeds from PIR and RIB withstood freezing, they would probably not withstand it much longer: with longer storage time, these seeds would also die. The reason is that electrical conductivity had already been significantly altered. Thus, although less intensely, membranes would already been ruptured and the embryos would probably lose viability in the subsequent periods. 


\section{CONCLUSIONS}

The conditions under which the Inga vera embryos had been determine their degree of maturation at the time of dispersion and, consequently, their tolerance to desiccation and freezing.

\section{ACKNOWLEDGEMENTS}

To Centro de Exposições Imigrantes, the City Council of Piracicaba, the Polo Regional Vale do Ribeira and the Universidade Federal de Lavras, for their permission and assistance in the collections; to the Vale do Ribeira Regional Hub, the Universidade de São Paulo and the Universidade Federal de Lavras, for providing meteorological data; to Dr. Lucia Rossi (Institute of Botany), for her collaboration and identification of the species; to CNPq and CAPES, for granting scholarships.

\section{REFERENCES}

ANDRÉO, Y.; NAKAGAWA, J.; BARBEDO, C.J. Mobilização de água e conservação da viabilidade de embriões de sementes recalcitrantes de ingá (Inga vera Will. subsp. affinis (DC.) T. D. Pennington). Revista Brasileira de Botânica, v.29, n.2, p.309-318, 2006. http://www. scielo.br/pdf/rbb/v29n2/a12v29n2.pdf

ARAUJO, A.C.F.B.; BARBEDO, C.J. Changes in desiccation tolerance and respiratory rates of immature Caesalpinia echinata Lam. seeds. Journal of Seed Science, v.39, n.2, p.123-132, 2017. https://www.scielo.br/scielo.php?pid=S231715372017000200123\&script=sci_arttext

BARBEDO, C.J.; CICERO, S.M. Utilização do teste de condutividade elétrica para previsão do potencial germinativo de sementes de ingá. Scientia Agricola, v.55, n.2, p.249-259, 1998. https://www.scielo.br/scielo.php?script=sci_arttext\&pid=S010390161998000200013\&lng=pt\&tlng=pt

BARBEDO, C.J. A new approach towards the so-called recalcitrant seeds. Journal of Seed Science, v.40, n.3, p. 221-236, 2018. https://www.scielo.br/pdf/jss/v40n3/2317-1545-jss-40-03-221.pdf

BILIA, D.A.C.; BARBEDO, C.J.; CICERO, S.M.; MARCOS-FILHO, J. Ingá: uma espécie importante para recomposição vegetal em florestas ripárias, com sementes interessantes para a ciência. Informativo ABRATES, v.13, p.26-30, 2003.

BONJOVANI, M.J.; BARBEDO, C.J. Respiration and deterioration of Inga vera ssp. affinis embryos stored at different temperatures. Journal of Seed Science, v.41, n.1, p.44-53, 2019. https://www.scielo.br/pdf/jss/v41n1/2317-1545-jss-41-01-44.pdf

BONJOVANI, M.J.; BARBEDO, C.J. Sementes recalcitrantes: intolerantes a baixas temperaturas? Embriões recalcitrantes de Inga vera Willd. subsp. affinis (DC.) T.D. Penn. toleram temperatura subzero. Revista Brasileira de Botânica, v.31, n.2, p.345-356, 2008. https://www.scielo.br/pdf/rbb/v31n2/v31n2a17.pdf

BONJOVANI, M.R.; BARBEDO, C.J. Induction of tolerance to desiccation and to subzero temperatures in embryos of recalcitrant seeds of inga. Journal of Seed Science, v.36, n.4, p.419-426, 2014. https://www.scielo.br/pdf/jss/v36n4/a06v36n4.pdf

BONJOVANI, M.R.; BARBEDO, C.J. Respiração de embriões recalcitrantes de Inga vera Willd. subsp. affinis (DC.) T.D. Pennington sob diferentes temperaturas, potenciais osmóticos e aplicação de ABA. Hoehnea, v.47, e012020, 2020. https://www.scielo.br/pdf/ hoehnea/v47/2236-8906-hoehnea-47-e012020.pdf

BRASIL. Ministério da Agricultura, Pecuária e Abastecimento. Regras para análise de sementes. Ministério da Agricultura, Pecuária e Abastecimento. Secretaria de Defesa Agropecuária. Brasília: MAPA/ACS, 2009. 399p. https://www.gov.br/agricultura/pt-br/ assuntos/insumos-agropecuarios/arquivos-publicacoes-insumos/2946_regras_analise_sementes.pdf

BRAZ, M.S.S.; FREITAS, S.L.S.; CAMPOS, M.A.L.; MIRANDA, D.D.O.A.; COSME, M.C. Caracterização morfológica do fruto, semente, plântula e planta jovem e germinação de Inga ingoides (Rich) Willd. Cerne, v.18, n.3, p.353-360, 2012. https://www.scielo.br/pdf/ cerne/v18n3/a01v18n3.pdf 
CACCERE, R.; TEIXEIRA, S.P.; CENTENO, D.C.; FIGUEIREDO-RIBEIRO, R.C.L.; BRAGA, M.R. Metabolic and structural changes during early maturation of Inga vera seeds are consistent with the lack of a desiccation phase. Journal of Plant Physiology, v.170, n.9, p.791-800, 2013. http://www.sciencedirect.com/science/article/pii/S0176161713000199

DAWS, M.I.; CLELAND, H.; CHMIELARZ, P.; GORIN, F.; LEPRINCE, O.; MATTHEWS, S.; MULLINS, C.E.; THANOS, C.A.; VANDVIK, V.; PRITCHARD, H.W. Variable desiccation tolerance in Acer pseudoplatanus seeds in relation to developmental conditions: a case of phenotypic recalcitrance? Functional Plant Biology, v.33, n.1, p.59-66, 2006. http://www.publish.csiro.au/paper/FP04206.htm

FAO. Genebank standards for plant genetic resources for food and agriculture. Roma: FAO, 2014. http://www.fao.org/home/en/

FONSECA, S.C.L.; FREIRE, H.B. Sementes recalcitrantes: problemas na pós-colheita. Bragantia, v.62, n.2, p.297-303, 2003. https:// www.scielo.br/pdf/brag/v62n2/v62n2a16.pdf

GOLDFARB, M.; CAVALCANTI-MATA, M.E.R.M.; DUARTE, M.E.M. Crioconservação de sementes oleaginosas. Revista Brasileira de Produtos Agroindustriais, v.19, n.1, p.113-121, 2017. http://www.deag.ufcg.edu.br/rbpa/rev191/rev19114.pdf

GUARDIA, M.C.; ASPERTI, L.M.; CANCIAN, G.M.; BARBEDO, C.J. Tolerância à dessecação e armazenamento de sementes de Myrcianthes pungens (O. Berg) D. Legrand (Myrtaceae). Hoehnea, v.47, e192020, 2020. https://www.scielo.br/pdf/hoehnea/ v47/2236-8906-hoehnea-47-e192020.pdf

HELL, A.F.; KRETZSCHMAR, F.S.; SIMÕES, K.; HEYER, A.G.; BARBEDO, C.J.; BRAGA, M.R.; CENTENO, D.C. Metabolic changes on the acquisition of desiccation tolerance in seeds of the Brazilian native tree Erythrina speciosa. Frontiers in Plant Science, v.10, article 1356, 2019. https://www.frontiersin.org/articles/10.3389/fpls.2019.01356/full

KADER, A.A.; SALTVEIT, M.E. Respiration and gas exchange. In: BARTZ, J.A.; BRECHT, J.K.; WEICHMANN, J. (ed.). Postharvest physiology and pathology of vegetables. New York: Marcel Deckker, 2002. p.7-29.

LAMARCA, E.V.; BARBEDO, C.J. Sensibilidade à dessecação de embriões de Inga vera willd. formados sob diferentes condições ambientais. Revista Árvore, v.39, n.6, p.1083-1092, 2015. https://www.scielo.br/pdf/rarv/v39n6/0100-6762-rarv-39-06-1083.pdf

LAMARCA, E.V.; BARBEDO, C.J. Short storability of Caesalpinia echinata seeds as a consequence of oxidative processes. Hoehnea, v.39, n.4, p.577-586, 2012. https://www.scielo.br/pdf/hoehnea/v39n4/06.pdf

LAMARCA, E.V.; BONJOVANI, M.R.; FARIA, J.M.R.; BARBEDO, C.J. Germinação em temperatura sub-ótima de embriões de Inga vera subsp. affinis obtidos sob diferentes condições ambientais. Rodriguésia, v.64, n.4, p.877-885, 2013. https://www.scielo.br/pdf/rod/ v64n4/v64n4a15.pdf

LAMARCA, E.V.; PARISI, J.J.D.; BARBEDO, C.J. Taxas respiratórias de sementes de Eugenia pyriformis Cambess. dispersas em diferentes estádios de maturação. Hoehnea, v.47, e1012019, 2020. https://www.scielo.br/pdf/hoehnea/v47/2236-8906-hoehnea47-e1012019.pdf

LEPRINCE, O.; BUITINK, J.; HOEKSTRA, F.A. Axes and cotyledons of recalcitrante seeds of Castanea sativa Mill. exhibit contrasting responses of respiration to drying in relation to desiccation sensitivity. Journal of Experimental Botany, v.50, n.338, p.1515-1524, 1999. https://academic.oup.com/jxb/article-abstract/50/338/1515/509819

MARCOS-FILHO, J. Fisiologia de sementes de plantas cultivadas. 2ed. Londrina: ABRATES, 2015. 660p.

MATA, M.F.; SILVA, K.B.; BRUNO, R.L.A.; FELIX, L.P.; MEDEIROS-FILHO, S.; ALVES, E.U. Physiological maturity of ingazeiro Inga striata Benth. seeds. Semina: Ciências Agrárias, v.34, n.2, p.549-566, 2013. http://www.uel.br/revistas/uel/index.php/semagrarias/article/ view/9327

MAYRINCK, R.C.; VILELA, L.C.; PEREIRA, T.M.; RODRIGUES-JUNIOR, A.G.; DAVIDE, A.C.; VAZ, T.A.A. Seed desiccation tolerance/ sensitivity of tree species from Brazilian biodiversity hotspots: considerations for conservation. Trees, v.33, p.777-785, 2019. https://link.springer.com/article/10.1007/s00468-019-01815-8

PARISI, J.J.D.; BIAGI, J.D.; BARBEDO, C.J.; MEDINA, P.F.; LAMARCA, E.V. Respiratory rates of Inga vera Willd. subsp. affinis (DC.) T.D. Penn. seeds. Floresta e Ambiente, v.26, n.4, e20171033, 2019. https://www.scielo.br/pdf/floram/v26n4/2179-8087-floram26-04-e20171033.pdf

PARISI, J.J.D.; BIAGI, J.D.; MEDINA, P.F.; BARBEDO, C.J. Fungicide and drying effects on the viability of recalcitrant seeds of Inga vera subsp. affinis. Tropical Plant Pathology, v.41, p.177-182, 2016. https://link.springer.com/article/10.1007/s40858-016-0084-6 
PEREIRA, L.C.V.; MAYRINCK, R.C.; ZAMBON, C.R.; JOSÉ, A.C.; FARIA, J.M.R. Storage of short-lived seeds of Inga vera subsp. affinis in osmotic medium. Seed Science Research, v.30, n.2, p.156-160, 2020. https://www.cambridge.org/core/journals/seed-scienceresearch/article/storage-of-shortlived-seeds-of-inga-vera-subsp-affinis-in-osmotic-medium/280F58459A4226A536ECFBB1A9FB57CE

SANTANA, D.G.; RANAL, M.A. Análise da germinação: um enfoque estatístico. Brasília: Universidade de Brasília, 2004.

SCHULZ, D.G.; ORO, P.; VOLKWEIS, C.; MALAVASI, M.M.; MALAVASI, U.C. Maturidade fisiológica de morfometria de sementes de Inga laurina (Sw.) Willd. Floresta e Ambiente, v.21, n.1, p.45-51, 2014. https://www.scielo.br/pdf/floram/v21n1/aop_floram_054713.pdf

SILVA, E.A.A.; OLIVEIRA, J.M.; PEREIRA, W.V.S. Fisiologia das sementes. In: BARBEDO, C.J.; SANTOS-JUNIOR, N.A. (org.). Sementes do Brasil: produção e tecnologia para espécies da flora brasileira. São Paulo: Instituto de Botânica, 2018. p.15-40.

VERTUCCI, C.W.; FARRANT, J.M. Acquisition and loss of desiccation tolerance. In: KIGEL, J.; GALILI, G. (ed.). Seed development and germination. New York: Marcel Dekker, 1995. p.237-271.

VILLA NOVA, N.A.; PEDRO-JÚNIOR, M.J.; PEREIRA, A.R.; OMETTO, J.C. Estimativa de graus-dia acumulados acima de qualquer temperatura base em função das temperaturas máxima e mínima. Caderno Ciência da Terra, v.30, p.1-8, 1972.

WALTERS, C. Orthodoxy, recalcitrance and in-between: describing variation in seed storage characteristics using threshold responses to water loss. Planta, v.242, p.397-406, 2015. http://link.springer.com/article/10.1007\%2Fs00425-015-2312-6

WALTERS, C.; BERJAK, P.; PAMMENTER, N.; KENNEDY, K.; RAVEN, P. Preservation of recalcitrant seeds. Science, v.339, n.6122, p.915916, 2013. http://science.sciencemag.org/content/339/6122/915 use, distribution, and reproduction in any medium, provided the original work is properly cited. 